\title{
EEG spectra in dyslexic and normal readers during oral and silent reading *
}

\author{
D. Galin ", J. Raz ${ }^{\text {b }, ~ G . ~ F e i n ~}{ }^{\text {a.c }}$, J. Johnstone ", J. Herron c and C. Yingling a \\ "Langley Porter Psychiatric Institute. Unicersity of California, San Francisco, C.A (U.S.A.). "School of Public Health, Unitcrity of Michigan, Ann \\ Arbor. MI (U.S.A.), "San Francisco VA Medical Center, San Francisco, CA (U.S.A.), \\ "Neurodata Inc., Pasadena, CA (U.S.A.), and" (alifornia Neuropsychology Serites, San Rafael, CA (U.S.A.)
}

(Accepted for publication: 13 August 1991)

\begin{abstract}
Summary EEGs of extensively screened dyslexics and normal readers were recorded while they read easy and difficult texts silently and orally, and during two other verbal tasks which also differed in overt speaking but had no reading component: narrative speaking and listening to a story. Mid-temporal, central and parietal leads were referenced to linked ears and to ( $z$. Large differences between tasks and between groups were found.

With the linked ears reference, power was higher in all bands in oral reading than in silent reading, with the largest change occurring in the temporal leads. In the theta and low beta bands the difference between oral and silent reading was greater for controls than for dyslexics. These effects were not accounted for by differences in reading speed or in difficulty. Similar results were found in two cohorts of subjects.

The difference between groups in theta was found only in the reading tasks. In contrast, the group difference in low beta was also found in the change from listening to speaking. This implies that the oral-silent group difference in theta is related to some aspect of the reading tasks other than the presence or absence of overt speaking, and that the low beta group difference is related to some aspect of overt speaking rather than to reading per sc. With the $\mathrm{Cz}$ reference no group differences were found.

It is suggested that the groups differ in the reading strategies they use, and the degree to which they shift strategy between the silent and oral 1asks. We hypothesize that these cognitive differences are reflected in the theta activity from the temporal lobe.

While there were many differences between the tasks in alpha power and assymmetry, no group differences involving alpha were found.
\end{abstract}

Key words: EEG spectra; Dyslexia; Reading; Oral: Silent: Speaking: Theta: Beta: Lateralization

Numerous studies have used electrophysiological methods in attempts to discriminate dyslexic from normal readers and to identify brain mechanisms underlying specific reading disability. No consensus has emerged on whether there are EEG features characteristic of dyslexia (see reviews by Benton 1975; Hughes 1978; John et al. 1977; Fein et al. 1986; Duffy et al. 1988). There have been remarkably few reports of EEG recordings during reading, the actual behavior of interest (Sklar et al. 1973; Rebert et al. 1978; Leisman and Ashkenazi 1980; Duffy et al. 1980, 1988; Lubar and Deering 1981). We have recorded the EEG during

Correspondence to: David Galin, M.D., LPPI-Box (0844, University of California at San Francisco, San Francisco, CA 94143-(1)844 (U.S.A.).

\footnotetext{
* Many investigators have collaborated in this program, which included many aspects besides the EEG spectrum studies. In addition to the present authors, Brian Brown, Linda Davenport, Pat Lund, Susan Trout, and Marilyn Marcus were colleagues in this project. Supported in part by NICHD Contract N01-HD-8-2824, NINDCS Grant IROI-NS17657, and U.C.S.F. Academic Senate Research Committee. Thanks to California Assemblyman John Vasconcellos for his support and encouragement.
}

reading and found differences between dyslexics and the normal readers which were not found in the resting EEG, or during other active tasks.

Early EEG studies of dyslexics relied on clinical interpretation of recordings from passive subjects, and a high frequency of abnormal findings was often reported (Hughes 1978). However, Conners (1978) has pointed out that the type of abnormalities found differed from one study to another (e.g., epileptic spikes, slow waves), and that the diagnosis of dyslexia was often not well supported. He further noted that there was a correlation of -0.91 between the percentage of abnormalities found and the year in which the study was published and concluded that the high incidence of abnormalities found in early studies was due to inclusion of subjects who had problems other than reading disability.

The search for electrophysiological markers in dyslexia has continued, with investigators looking for more subtle abnormalities in patterns of EEG activation, in coherence, and in evoked potential features (Sklar et al. 1973; John et al. 1977; Bakker and Vinke 1985; Davidson et al. 1985; Duffy and McAnulty 1985). However, the problem of specificity remains; the clini- 
cal and theoretical significance of such findings depends on whether the abnormalities found are associated with learning disabilities per se, or whether they are secondary to other coexisting neurological deficits. A very sophisticated and extensive program was carried out by E.R. John and his colleagues, called "neurometrics" (John et al. 1977, 1980; Ahn et al. 1980). They applied the principles of numerical taxonomy to analysis of resting EEG and evoked potentials, using computerized feature extraction, a large normative data base, and an unusually large sample of learning disabled children. They reported that "neurometric EEG measures... discriminated between normal and learning disabled children better... than psychometric measures" (John et al. 1977). We followed up these interesting results by applying the neurometrics procedure to a highly screened group of severe dyslexics and a carefully matched control group, all free of overt neurological dysfunction. Unfortunately, it did not discriminate between these groups. We concluded that even severe dyslexia per se is not associated with the neurometric abnormalities reported in more heterogeneous learning disabled populations (Yingling et al. 1986). In further studies of the resting EEG in these highly screened dyslexics and controls (Fein et al. 1986), we did find significant differences in beta power, but there was considerable overlap between the groups. Low beta power is not specific to dyslexia; it has also been reported in hyperactive children (Callaway et al. 1983).

In addition to the resting EEG, our research program has emphasized recording while the subjects are actively engaged in the behavior of interest; the deficit in a system may only be detectable when the system is tested "under load." We have studied two groups of cognitive tasks, one focused on lateral specialization for verbal and spatial processing in general (e.g., narrative speech vs. block design construction), and the other involving reading per se. In the lateral specialization studies we found no support for the "Orton hypothesis" of disordered cerebral dominance as the basis for dyslexia (Galin et al. 1988). In the present paper we report our findings on the EEG during reading.

All subjects in this study met rigorous screening criteria designed to ensure no overlap in reading abilities between the two groups, and no coexisting neurological or psychological conditions which might cause EEG findings unrelated to dyslexia per se. We place great stress on this aspect of our work because we believe that the lack of consensus in the dyslexia literature is in part due to the wide variety of criteria for subject selection. Many studies have used heterogeneous samples of dyslexic children, without adequate control over potentially confounding demographic, sensory, neurological or psychometric variables. Handedness and sex have varied both within and between studies without being evaluated as controlled variables. Samples have included children with impaired sensory acuity, hyperactivity, emotional or psychiatric disorders, assorted gross neurological signs or below normal IQ. Furthermore, control subjects have often received much less screening than the "experimental" group. All of these factors have been controlled in the present study.

\section{Methods}

Subjects

Fifty-six dyslexics and 57 normal readers were selected. We chose a 2-cohort design in which the second cohort was used to cross-validate results from the first cohort. The first cohort consisted of 34 dyslexic and 35 controls, age 10-12. The second cohort consisted of 22 dyslexics and 22 controls, age 9-13. All were righthanded Caucasian boys, middle class, whose only language was English, and who had attended school regularly. Most of the dyslexics were referred from private remedial schools and other special education programs. Most of the controls were self-referred in response to newspaper ads. To avoid a skewed self-selection of controls, we solicited boys to participate in a "scientific research" project, with no mention of reading.

Parent interviews were conducted to establish normal pregnancy, birth and neonatal health, and a history free from accidents or illness which might have affected brain function. Children with a history of hyperactivity, epilepsy, emotional problems, developmental anomalies, or previously diagnosed deficits in oral language were excluded. Candidates were given clinical hearing, vision, and neurological examinations (Touwen and Prechtl 1970), the Wechsler Intelligence Scale for Children (Revised) (Wechsler 1974), and oral and silent reading tests. Candidates with Full-Scale IQs below 88, or with positive findings on the sensory or neurological tests, were excluded (see Johnstone et al. 1984 for detailed listing of exclusion criteria).

Reading ability was considered in relation to what would normally be expected on the basis of a child's age and IQ. Oral reading was tested with the Gray Oral Reading Test (Gray 1963), and silent reading vocabulary and comprehension were tested with the Gates-MacGinitie Reading Tests (Gates and MacGinitie 1965). A reading quotient (Myklebust 1968) was used to determine the ratio of actual achievement to expected achievement:

Reading quotient $=$

$[(2 \times$ reading age $) /($ chron , age + mental age $)]$. 


\section{TABLE I}

Age, IQ, and reading scores of dyslexics and controls (cohort 1 and cohort 2 combined).

\begin{tabular}{lrrrrr}
\hline & \multicolumn{2}{c}{ Dyslexics $(\mathrm{n}=49)$} & & \multicolumn{2}{c}{ Controls $(\mathrm{n}=51)$} \\
\cline { 2 - 3 } & Mean & S.D. & & Mean & S.D. \\
\hline Age (months) & 138.2 & $(13.4)$ & & 140.6 & $(11.4)$ \\
Verbal IQ & 100.2 & $(9.5)$ & & 112.8 & $(10.5)$ \\
Performance IQ & 109.1 & $(12.0)$ & & 111.7 & $(11.5)$ \\
Full scale IQ & 104.7 & $(10.0)$ & & 113.7 & $(10.9)$ \\
Oral reading grade & 2.0 & $(0.5)$ & & 8.6 & $(2.3)$ \\
Reading comp. grade & 3.1 & $(1.3)$ & & 9.2 & $(2.4)$ \\
\hline
\end{tabular}

Mental age was based on WISC-R Fullscale IQ. All dyslexics accepted into the study had reading quotients at or below 0.80 on both reading tests, which is equivalent to 2 years below grade level for a 10 -year-old child with an IQ of 100 . All control subjects' reading quotients on the Gates-MacGinitie were 0.93 or above, and their oral reading scores were within 1 year of actual grade placement, or higher.

These stringent screening criteria resulted in the exclusion of several hundred candidates. Rejection rates were roughly equal for both dyslexic and control groups. Even among those who had passed the telephone interviews, less than one-third met all the psychometric, neurological, and sensory criteria. The groups finally selected consisted of essentially "pure" dyslexics, and good to superior readers, with equivalent group means and distributions of age and Performance IQ. There was a 6.0 grade level difference between groups in reading comprehension and a 6.4 grade level difference in oral reading (see Table I).

\section{Procedures}

Subjects worked at an inclined table in a sound attenuated room monitored with video and intercom. EEG was recorded during passive conditions (resting, with eyes open and with eyes closed, 3 min each), and during active cognitive tasks, including block design, mirror tracing, oral and silent reading of easy and hard texts, listening to a story, narrative speech, and vigilance-reaction time. For the first cohort the series of tasks was repeated in reverse order to control for time trends. For the second cohort some conditions were dropped and the repetitions were omitted. Testing sessions began mid-morning and typically lasted $5 \mathrm{~h}$ including lunch and breaks. Great care was taken to maintain the subject's alertness and cooperation, including testing vigilance at the beginning and end of the session, and monitoring performance on each task. This report presents the results from the reading tasks, listening, and narrative speech. For a complete description of procedures, see Yingling and Galin (1982).

\section{Cognitive tasks}

\section{Oral and silent reading}

We recorded during both silent and oral reading because they differ in cognitive demands. To control for difficulty we used both easy and difficult reading materials selected for each child, 2 grade levels below and above his tested reading level. To get material which was truly easy for some dyslexics we had to go to primer levels, and to get hard enough material for some of the bright normal readers we had to go to college levels.

In order to minimize test anxiety we explained that this was not at all like a test in school. The children were advised that while some passages would be quite easy, we knew that others would be very, very, difficult for them, because we were studying what the brain does when it is working hard. They only had to try their best, even when they knew they were making mistakes, and there would be no grades. This seemed to be reassuring and we encountered no reluctance to participate from any subject.

The text to be read was presented on double-spaced typed pages on the work table. Each task lasted 2-2.5 min. Questions were asked after each selection to further motivate attending and to verify their engagement in the silent tasks. These questions provided an informal measure of comprehension but were not equivalent to a standardized comprehension test. If performance was questionable, new materials were presented. Reading speed in words per minute was scored.

\section{Narratice speech ("SPEAK")}

Subjects were instructed to choose a topic on which they could talk continuously for several minutes (favorite movies, TV shows, books or personal experiences). Visual fixation was maintained on the work table as for the READ tasks. The speech samples averaged $240 \mathrm{sec}$ (range 140-330 sec) and were recorded for later analysis (Davenport et al. 1985).

\section{Listening to a story ("LISTEN")}

The child listened to a 2 min tape-recorded factual account, with eyes open and fixed on the work table. Stories were taken from age-appropriate texts. Comprehension questions were asked after each story to verify the child's engagement with the task.

The order of the tasks was Read Silent Easy (RSE), Read Oral Easy (ROE), Listen to Story, Narrative Speech, Read Silent Hard (RSH), and Read Oral Hard $(\mathrm{ROH})$. Subjects were coached to eliminate extraneous facial movements and relax the neck and jaw in order to minimize EMG contamination. 


\section{EEG recording and signal processing procedures}

EEG was recorded during each task using Grass gold cup electrodes from bilateral central (C3, C4), parietal (P3, P4), mid-temporal ( $\mathrm{T}_{\text {mid L }}$ located halfway between $\mathrm{T} 3$ and $\mathrm{T} 5$, and $\mathrm{T}_{\text {mid } \mathrm{R}}$ located halfway between $\mathrm{T} 4$ and $\mathrm{T} 6$ ), and linked ear (A1A2) leads, all referenced to vertex $(\mathrm{Cz})$. A separate montage referenced to linked ears was derived off-line by subtracting the A1A2-Cz channel from the other leads. We used the $\mathrm{Cz}$ reference as well as the more common linked ears reference because we have found it to be more sensitive to hemispheric asymmetries. Eye movements were monitored by electrodes placed above and medial, and below and lateral to the left eye. Signals were amplified with a Grass Model 7 polygraph, with Model 7P5 preamplifiers set to half amplitude cut-offs of 0.3 and $35 \mathrm{c} / \mathrm{sec}$. The EEG was digitized by a NOVA minicomputer at 256 points/sec and stored on magtape. An $80 \mu \mathrm{V} 10 \mathrm{c} / \mathrm{sec}$ calibration signal was recorded on each channel both before and after the session. Off-line, each 1 sec epoch of digitized data was visually edited for movement, EOG, EMG, and other artifact. Each accepted epoch was then subjected to Fast Fourier Transform (FFT), calibrated to actual $\mu V^{2}$ in $1 \mathrm{c} / \mathrm{sec}$ bins, and stored on floppy disks. Means and standard deviations were computed for each task, for each subject. Sample lengths averaged 2-2.5 min for each task (minimum length accepted $=30 \mathrm{sec}$ ) Data were banded into delta $(0.5-3.5 \mathrm{c} / \mathrm{sec})$, theta $(3.5-7.5$ $\mathrm{c} / \mathrm{sec})$, alpha $(7.5-13.5 \mathrm{c} / \mathrm{sec})$, low beta $(13.5-20.5$ $\mathrm{c} / \mathrm{sec})$ and high beta $(20.5-32.5 \mathrm{c} / \mathrm{sec})$.

\section{Data analysis}

Statistical analysis was performed on the common logarithms of the absolute spectral power. In the first analysis a repeated measures ANOVA was performed for each reference and band using "Readtype" (oral vs. silent), "Difficulty" (easy vs. hard), "Lead-pair" (midtemporal, central, and parietal), and "Side" (left vs. right) as within-subject factors. The between-subject factor was Group (dyslexic vs. control). The sphericity assumption of the ANOVA was applicable only to effects involving lead-pair, since the other factors have only two levels. The Huynh-Feldt correction (Huynh and Feldt 1976) was applied to the computation of significance probabilities for these effects. To follow up these results a second ANOVA was performed on TALKING vs. NO TALKING tasks: Read Oral and Speaking vs. Listening and Read Silent. This analysis is detailed below.

In addition to considering significance levels, we computed the size of effects, expressed in standard deviation units, using a generalization of the procedure for matched-pairs $t$ tests (equation 2.35, p. 48, Cohen
1977). The quantitative measure of effect size adds perspective to results reported only with $P$ values. Significance levels depend on sample size and give no information about the magnitude of the differences. Effect sizes of 0.5 S.D. are considered medium, whereas effect sizes of 0.8 S.D. are large and obvious, e.g., "the difference in mean IQ between Ph.D.s and college freshmen" (Cohen 1977).

Because of the large number of significance tests computed we applied a Bonferroni correction. With 210 tests (21 main effects and 2- and 3-way interactions, for 5 bands with 2 references), the alpha level equivalent to $P=0.05$ for a single test would be $0.05 / 210=0.00024$. Since we had replication data from 2 independent cohorts, we combined their results using the meta-analytic techniques of Rosenthal (1984), which establish a new alpha level for the sum or product of the $P$ values in the 2 cohorts corresponding to Bonferroni-corrected levels for each cohort alone. By this method an effect was considered significant when the sum of $P$ values for both cohorts was less than 0.022 .

Significant effects were investigated in more detail. We tested for possible regional localization by computing effect sizes for each lead. Plots of the distribution of oral minus silent difference scores were examined to see if the group effects could be due to the influence of a small number of outliers. Reading speed was introduced as a covariate. Finally, plots of the difference scores and effect sizes were examined in $1 \mathrm{c} / \mathrm{sec}$ frequency bins, as well as broad bands.

\section{Results}

The basic data consisted of the log power in $1 \mathrm{c} / \mathrm{sec}$ bins from 1 to $32 \mathrm{c} / \mathrm{sec}$ for each lead and reference, for each task, for each subject. The major results concern the theta, alpha, and low beta bands with the linked-ears reference; means and S.D.s are presented in Tables II, III and IV. Full tables for all bands and both references are available on request. Cohorts I and II are presented separately in each table. In addition to the 6 active conditions, the eyes-open and eyes-closed resting conditions presented previously (Fein et al. 1986) are included for comparison (eyes-open condition not tested in cohort II).

\section{(I) ANOVA on reading tasks}

The first analysis focused on the reading tasks. Ten separate repeated measures ANOVAs were performed on log power, one for each reference and band, with GROUP (Dyslexic vs. Control) as a between-subjects factor, and 4 within-subject factors: READTYPE (oral vs. silent), DIFFICULTY (easy vs. hard), LEAD-PAIR (mid-temporal, central, and parietal), and SIDE (left 


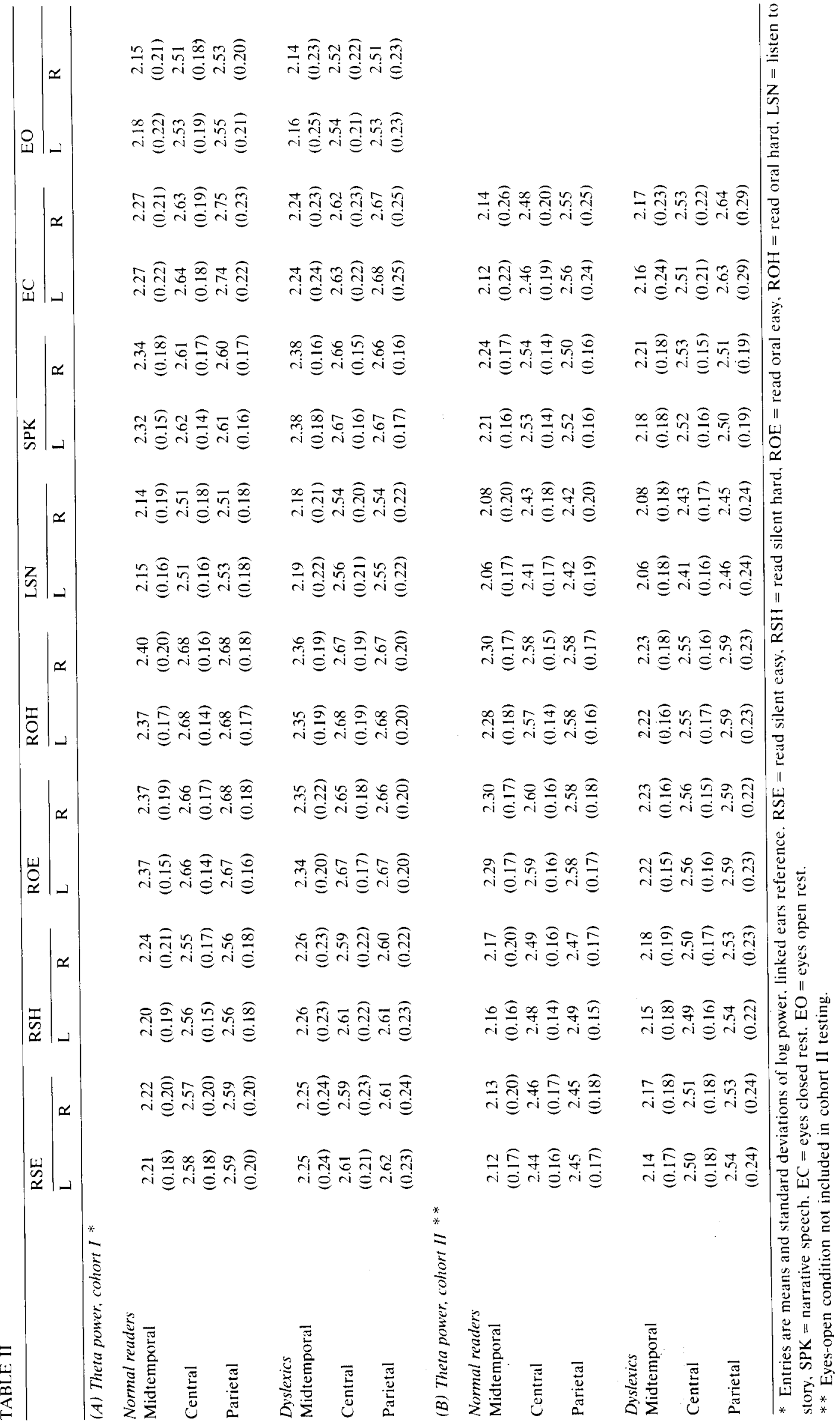




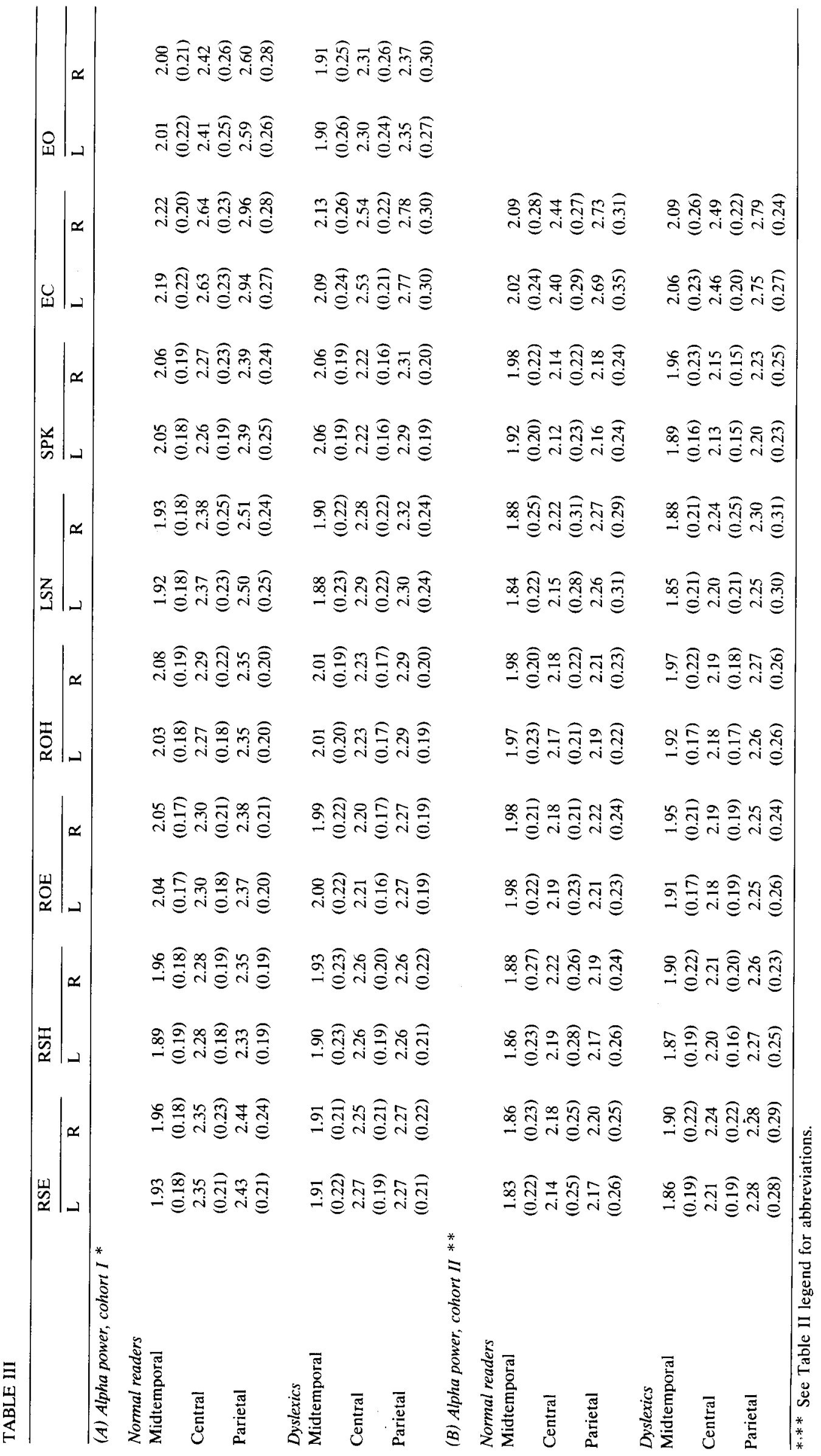




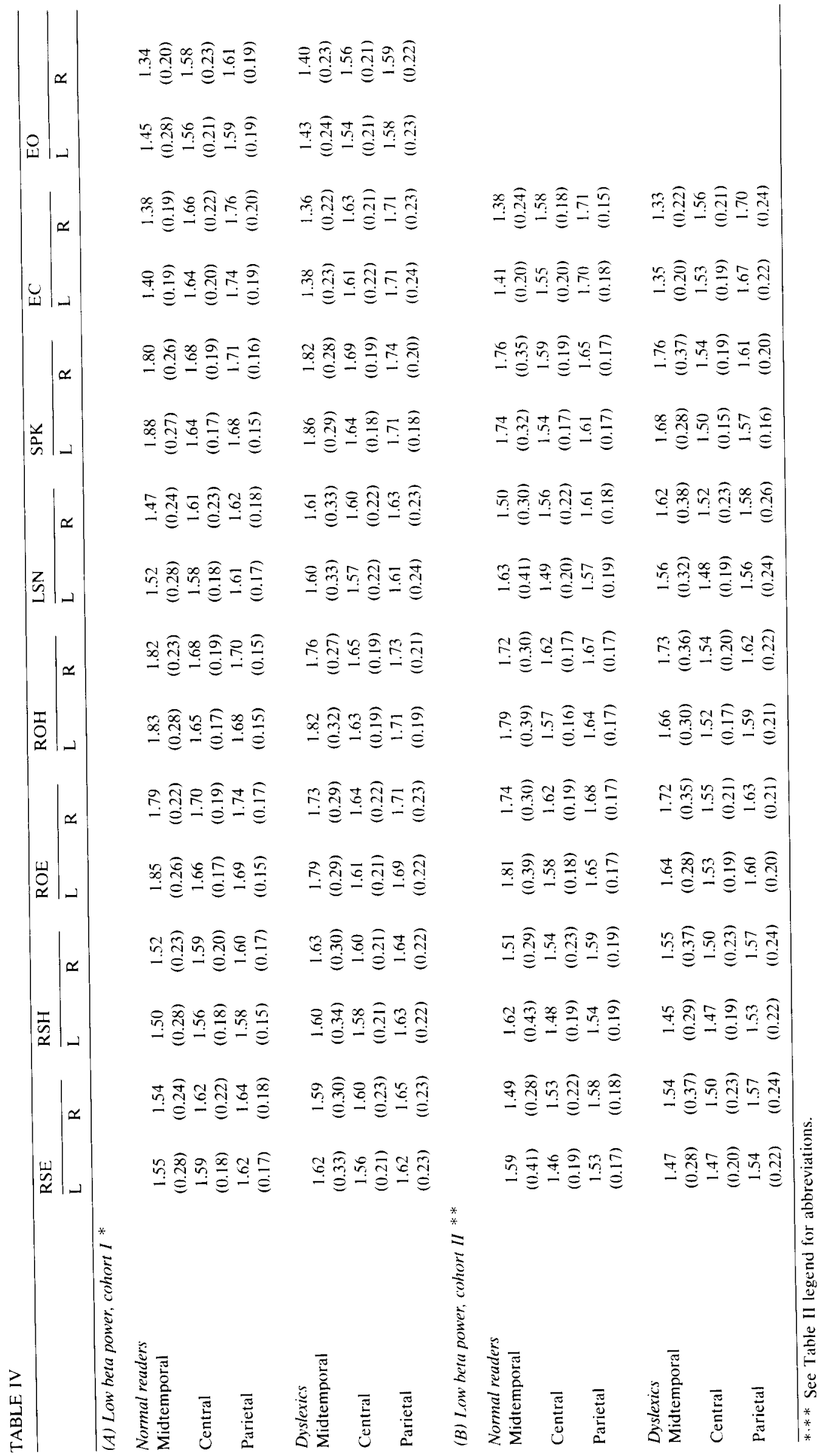


vs. right). Only effects which were significant over both cohorts with the Bonferroni correction are reported here. There were no significant main effects or consistent interactions with DIFFICULTY, and therefore data are presented for easy and hard conditions averaged, except as mentioned. The following abreviations will be used: Read Silent Easy (RSE), Read Silent Hard (RSH), Read Oral Easy (ROE), Read Oral Hard (ROH).

\section{(A) Effects unrelated to group}

(1) READTYPE: power is higher in oral than in silent reading. Large main effects of READTYPE were found in all bands. Power was higher during oral reading than during silent reading, except for theta and alpha with the $\mathrm{Cz}$ reference.

(a) Linked-ears reference. Power in all bands increased from silent to oral reading, $P<0.00001$ for each band, both cohorts, except alpha $P=0.02$ in cohort $\mathrm{I}, P=0.001$ in cohort II.

For each band a significant interaction of READTYPE by LEAD-PAIR was found for both cohorts $(P<0.00001$, except for delta cohort I $P=0.006)$. In each band the interaction was due to the READTYPE effect being largest in the mid-temporal leads. Alpha power increased slightly in the temporals and decreased slightly in central and parietal leads. These changes with READTYPE were symmetrical; there was no significant interaction with SIDE in any band for either cohort.

(b) $\mathrm{Cz}$ reference. Power in delta, low beta and high beta increased significantly from silent to oral, $P<$ 0.00007 , both cohorts. For the theta band there was no change in power in either cohort, $P=0.62, P=0.72$. For the alpha band, power decreased most prominently in the central leads $(P<0.00001$ in cohort $\mathrm{I}, P<0.0005$ in cohort II).

A significant interaction of READTYPE by LEAD-PAIR was found for each band except theta $(P<0.00001$ for all bands, both cohorts, except delta cohort II $P=0.003$ ). For delta, low beta, and high beta, the interaction was due to the increase in power during oral reading being largest in the mid-temporal leads. In the alpha band the decrease during oral reading was most prominent in the central leads, with little change in the temporals. These changes with READTYPE were symmetrical: there was no significant interaction with SIDE in any band for either cohort.

(2) SIDE: asymmetry shows little relation to other variables. There was a significant main effect of SIDE with the $\mathrm{Cz}$ reference only, for the alpha band (right greater than left; cohort I $P=0.0001$, cohort II $P=$ $0.03)$. For low beta the effect was not quite significant; cohort I $P=0.0002$, cohort II $P=0.06$. The sum of $P$ values for the two cohorts $(0.060)$ was close to, but

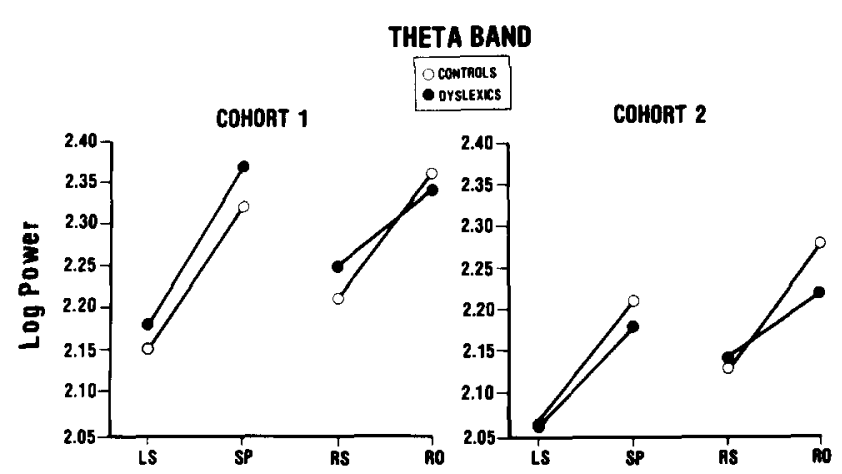

Fig. 1. Log power for theta band, left mid-temporal lead, linked ears reference. $\mathrm{LS}=$ listening to story, $\mathrm{SP}=$ narrative speaking, $\mathrm{RS}=$ reading silently, easy text, RO = reading orally, easy text. Note: the plotted values are group means, which include between-subject variance. This obscures to some extent the very consistent within-subject task differences which are revealed by the ANOVA within-subjects repeated measures design.

exceeded, the Bonferroni-corrected criterion value (0.022). There were no significant interactions of SIDE with GROUP, READTYPE, or LEAD-PAIR.

\section{(B) Effects related to group}

(1) Differences between dyslexics and controls in oral and silent reading: interactions of GROUP with READTYPE. The ANOVA showed no main effects of GROUP for any band, for either cohort or reference. However, with the linked-ears reference, there were significant interactions of GROUP-by-READTYPE; while power increased from silent to oral reading for both groups, the change in power between tasks was significantly smaller for dyslexics than for normal readers. This interaction involved frequency bands which we did not expect; theta, low beta, and marginally significant for high beta. There was no significant interaction in the alpha band. Similar results were found in both cohorts. The size of the effects were quite large, ranging up to 1.23 standard deviation units (Cohen 1977) depending on lead, band, and cohort. Note that these results were for differences between tasks within subject; in Figs. 1 and 2 and in Tables II, III, and IV group means for each task are shown, which do not

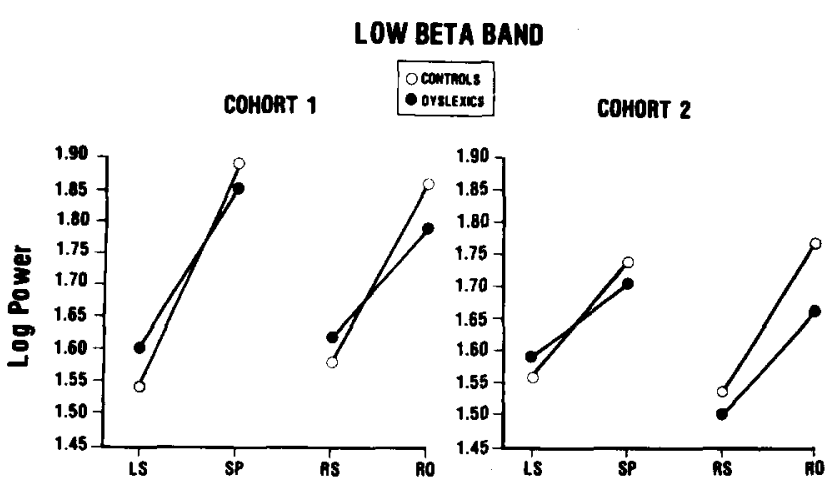

Fig. 2. Log power for low beta band. See legend tor Fig. 1. 
highlight the small but very consistent differences within subject. In other words, the variance of the within-subject task differences is small compared to the task differences. In the repeated measures analysis the larger between-subject variance is removed.

(a) Theta band (see Table II): the group means for theta log power, combining Easy and Hard, and pooling leads were:

\begin{tabular}{|c|c|c|c|c|c|c|c|}
\hline & & RS & RO & & & RS & $\mathrm{RO}$ \\
\hline \multirow[t]{2}{*}{ Cohort I } & Control & 2.45 & 2.58 & Cohort II & Control & 2.37 & 2.48 \\
\hline & Dyslexic & 2.47 & 2.55 & & Dyslexic & 2.40 & 2.46 \\
\hline
\end{tabular}

Note that there was no significant difference between dyslexics and controls for either RS or RO, only for the change from RS to RO. The $P$ value of the GROUP by READTYPE interaction for cohort I was $P=0.017$, effect size $=0.53$; for cohort II $P=0.0001$, effect size $=1.23$. These $P$ values jointly are well below the level necessary for significance with the Bonferroni correction. Examination of frequency histograms of individual subjects' RO-RS difference scores confirmed that the effect was not due to outliers. For the $\mathrm{Cz}$ reference the interaction was not significant for either cohort.

There were no significant 3- or 4-way interactions of GROUP by READTYPE with LEAD, SIDE, or DIFFICULTY in either cohort. In other words, the theta band GROUP by READTYPE effect was not systematically larger in the central, mid-temporal, or parietal region, or on one side or the other, or with easier or harder task demands.

To investigate the theta band GROUP by READTYPE interaction in more detail, effect size was computed in $1 \mathrm{~Hz}$ bins, for the left temporal lead and for all leads averaged (cohort $\mathrm{I}$, linked-ears reference). Effect size in both analyses was greatest at 4 and $5 \mathrm{~Hz}$ ( $\mathrm{ES}=0.65-0.9)$.

(b) Low beta band (see Table IV): the group means for low beta log power, combining Easy and Hard, and pooling leads, were:

\begin{tabular}{llllllll}
\hline & & RS & RO & & RS & RO \\
\hline Cohort I & Control & 1.58 & 1.73 & Cohort II & Control & 1.54 & 1.69 \\
& Dyslexic & 1.62 & 1.71 & & Dyslexic & 1.52 & 1.61 \\
\hline
\end{tabular}

As with the theta band, there was no significant difference between dyslexics and controls for either RS or RO, only for the change from RS to RO. The $P$ value of the GROUP by READTYPE interaction for cohort I was $p=0.0031$, effect size $=0.62$; for cohort II $P=0.014$, effect size $=0.83$. These $P$ values jointly are well below the level necessary for significance with the Bonferroni correction. Examination of frequency histograms of individual subjects' RO - RS difference scores confirmed that the effect was not due to out- liers. For the $\mathrm{Cz}$ reference the interaction was not significant when the $P$ values were corrected ( $P=0.05$, $0.06)$.

There were no significant 3- or 4-way interactions of GROUP by READTYPE with LEAD, SIDE, or DIFFICULTY in either cohort. In other words, the low beta GROUP by READTYPE effect was not systematically larger in the central, mid-temporal, or parietal region, or on one side or the other, oi with easier or harder task demands.

Analysis of effect size in $1 \mathrm{c} / \mathrm{sec}$ bins showed that the effect size in the low beta band was greatest at the lower end of the band, $14-17 \mathrm{c} / \mathrm{sec}$, and was near zero up to $24 \mathrm{c} / \mathrm{sec}$. Similar patterns were found for the left temporal lead and all leads averaged. Since any tonic EMG contamination remaining after editing would be maximum in the higher frequency bins, we conclude that the low beta effect is not an artifact of EMG activity.

(2) Possible determinants of the GROUP by READTYPE effect. Since a large and reliable EEG difference between dyslexics and controls appeared to be in some way related to a difference between silent and oral reading, we considered how these tasks differ. Some obvious features are difficulty, rate of processing words, and the requirement for speaking aloud in addition to decoding the text.

(a) Reading speed: we have used reading speed as an indirect index of difficulty and of the rate of processing words. An analysis of the reading speed data has been previously reported (Johnstone et al. 1984) and is repeated here briefly because it bears on the interpretation of the EEG spectra effects. For cohort I the mean and standard deviation for reading speeds (words/min) for each group and reading condition were as follows:

\begin{tabular}{lrrrr}
\hline \multicolumn{1}{l}{ RSE } & \multicolumn{1}{l}{ RSH } & \multicolumn{1}{l}{ ROE } & \multicolumn{1}{l}{ ROH } \\
\hline Controls & $193.0(61.0)$ & $145.2(47.1)$ & $146.4(26.2)$ & $100.1(20.8)$ \\
Dyslexics & $79.5(30.2)$ & $73.7(32.0)$ & $75.2(29.7)$ & $62.1(19.2)$ \\
\hline
\end{tabular}

Reading speeds for each group and task condition were normally distributed. Reading speed was analyzed with a repeated measures ANOVA: GROUP by DIFFICULTY by READTYPE. Aside from the expected main effects, there were interactions of GROUP by DIFFICULTY and GROUP by READTYPE. These were investigated with 2-sample and matched-pair $t$ tests. Controls showed significantly larger decrements from easy to hard reading than did dyslexics ( $F=39.72$, $P<0.0001)$. Controls slowed for difficult passages in both silent and oral reading (silent $t=7.50, P<0.0001$; oral $t=11.11, P<0.0001)$. Dyslexics slowed significantly only in oral reading $(t=3.21, P<0.003)$. Controls also showed a larger decrease from silent to oral reading than did dyslexics (GROUP by READTYPE: $F=23.37, \quad P<0.0001)$. Controls were significantly 
slower in oral than silent reading for easy as well as hard passages (easy $t=5.38, P<0.0001$, hard $t=7.26$, $P<0.0001$ ), whereas dyslexics read easy passages silently and aloud at the same speed, affected by READTYPE only with the hard passages $(t=2.62$, $P<0.01)$. Results were similar in cohort II.

The percentage of correct responses to the questions asked following each reading task provided only a rough index of comprehension. Nevertheless, the data may help in interpreting the reading speed and EEG results. Controls showed a large decrement in comprehension as well as speed on hard tasks, both silent and oral (silent $t=7.36, P<0.0001$, oral $t=5.87, P<$ 0.0001 . The dyslexics' comprehension decreased with difficulty only on the silent tasks (along with no significant slowing), and did not decrease significantly on the oral tasks (but with significant slowing).

We next examined the GROUP by READTYPE effects on theta and low beta power in relation to these reading performance data. Unfortunately, an analysis of covariance with reading speed partialled out of the GROUP by READTYPE effect was not possible, because speed was so highly correlated with group membership that the assumptions of ANCOVA were not met. However, simple inspection of the data indicates that reading speed by itself does not account for these effects. For example, in the RSE condition, dyslexics read at 79 words/min, controls at 193 words/min, more than twice the dyslexics' speed; if reading speed per se affected theta and beta power, then there should have been a large difference between groups for the RSE condition considered alone. In fact, the difference was not significant. Similarly, within the control group itself, speed decreased from 146 words / $\mathrm{min}$ in ROE to 100 words/min in $\mathrm{ROH}$, with no significant change in theta or beta power.

The ANOVA on spectral power showed no main effects or interactions for DIFFICULTY, for either group or for either type of reading, indicating that text difficulty by itself does not account for the EEG effects either. Therefore, we moved on to an analysis of the requirement for speaking aloud in addition to decoding the text.

\section{(II) ANOVA on tasks with and without talking}

Speaking aloud has cognitive aspects and also mechanical aspects, such as muscle activity and tongue movement. It is unlikely that the theta and low beta effects are due to the mechanical aspects. EMG contamination would be greatest in the higher frequency bins, and the size of our low beta effect was greatest at the low end of the band, from 14 to $17 \mathrm{c} / \mathrm{sec}$. Tongue movement artifacts could not account for the effects; they appear in only $5 \%$ of the population and are most prominent in the delta band (Klass and Bickford 1960).

\section{(A) TALK us. READ analysis}

Therefore, we devised another analysis to test whether the task and group differences in theta and beta power were related to some aspect of the reading process per se which is different in oral and silent reading, or due to the presence or absence of overt speech. We examined the EEG during two other verbal tasks that differ in overt speaking but have no reading component: narrative speaking and listening to a story. We performed a second repeated measures ANOVA on all the EEG variables from Listening to Speech (receptive, non-motor), Speaking (expressive, motor), Silent Reading (receptive, non-motor), Oral Reading (receptive and expressive, motor). For this analysis we used easy reading tasks only. The ANOVA used a "READING" factor (oral and silent reading vs. speaking and listening), a "TALKING" factor (oral reading and speaking vs. silent reading and listening), as well as GROUP, LEADPAIR (mid-temporal, parietal, and central), and SIDE. No Bonferroni correction was applied, since we made an a priori decision that only a few interactions were relevant, particularly READ by TALK by GROUP. The significance probabilities for the two cohorts were combined using the Rosenthal method described above. Rate of speech in narrative speaking was the same for both groups (Davenport et al. 1985).

With the $\mathrm{Cz}$ reference, in the theta band, there was no effect of the TALKING factor (i.e., no difference between Listening and Speaking, or between Read Silent and Read Oral), but a large effect of the READING factor (both Listening and Speaking lower than Read Silent and Read Oral). For the low beta band the results were reversed; a significant effect of TALKING, and no effect of READING. There were no effects involving group. The results were the same for both cohorts.

With the linked-ears reference, in both the theta and low beta bands, there was a main effect of TALK; power was higher in the tasks with talking than those without ( $P<0.00001$, both cohorts). Most important, in both bands there was a significant interaction of TALK with GROUP, and the interactions were different in the two bands. In low beta there was a 2-way TALK by GROUP effect; dyslexics showed a smaller increase in power than did the controls in both pairs of tasks, whether reading was involved or not. In theta there was a 3-way TALK by READ by GROUP effect; the dyslexics showed the same increase as the controls from Listen to Speak, but a significantly smaller increase than the controls from Read Silent to Read Oral. These results imply that (1) the oral-silent group difference in theta is related to some aspect of the 
reading tasks other than the presence or absence of overt speaking, and (2) the oral-silent group difference in low beta is related to some aspect of overt speaking rather than to reading per se.

These results were found in both cohorts. For theta, the $P$ value of the GROUP by READ by TALK interaction for the two cohorts combined as above was $<0.0001$, and for low beta the Group by Talk $P$ value was 0.008 . Figs. 1 and 2 show the group means for these tasks for the left mid-temporal lead.

\section{Discussion}

Recording during actual reading and other verbal tasks revealed unexpected differences between tasks and between groups. Power in all bands was higher during oral reading than during silent reading in both groups, but in the theta and low beta bands the change in power between tasks was significantly smaller for dyslexics than for normal readers. The difference between groups in theta was found only in the reading tasks. The group difference in low beta was also found in the change from listening to speaking as well as in the change from silent reading to oral reading and, therefore, seems to be related to some aspect of overt speech rather than to some aspect of reading. The effects were quite large, and similar results were found in both cohorts of subjects. These are the first large and replicable differences between the EEGs of dyslexics and normal readers which we have found.

Localization of effects: groups distinguished only with linked-ear reference

The group differences in theta were seen only with the linked-ears reference, not with $\mathrm{Cz}$. The effect size was not consistently greater in one lead than another (a mid-temporal advantage seen in the first cohort was not replicated), nor between left and right leads in each region. This pattern of results is consistent with the hypothesis that the signals critical to the group difference were being picked up by the linked-ears electrodes. The ear leads have been shown to be particularly sensitive to signals coming from the inferior temporal lobe by Sperling and Engel (1985). Their observation was made in the course of evaluating the efficiency of different electrode placements in detecting pathological epileptiform discharges from the temporal regions.

Unlike theta, low beta showed a significant Silent vs. Oral effect with both references, and with the $\mathrm{Cz}$ reference the low beta main effect of Side (power asymmetrical $\mathrm{R}>\mathrm{L}$ ) and interaction of group-by-task both approached significance. These differences in the pattern of results with the two references suggests that the theta and low beta effects may depend on separate mechanisms.

\section{Speculations on underlying cognitice mechanisms}

We will understand dyslexia better when we know more about normal reading. In spite of encouraging recent progress (e.g., Coltheart et al., 1980; Just and Carpenter 1986), we still know very little in neuropsychological terms about how normal children learn to read, or how skilled reading is done by normal adults. Reading is a relatively recent accomplishment on the list of human abilities; it has not been subjected to the prolonged evolutionary pressure which has shaped the neurological basis of speech. Therefore there may be many different cognitive strategies for reading, and thus more ways for it to break down.

Since the theta effect was an interaction between GROUP and READTYPE we must ask in what ways silent and oral reading might differ more for normal readers than for dyslexics. They obviously differ in the requirement for speaking aloud, in speed, and in difficulty; we have examined these variables (see Results above) and found that they did not account for the theta effect.

We propose another explanation post hoc, which will require further experiment to verify. We suggest that there are two critical differences between tasks, the requirement for pronouncing each word, and the requirement for understanding. Because of these differences the tasks are susceptible to different cognitive strategies. We suggest that the normal readers can use cither strategy and do switch, and that the dyslexics cannot.

Oral and silent reading differ in their customary purposes. The primary goal in oral reading is to pronounce each and every word to convey it literally to someone else, for them to assimilate it. Understanding is secondary. It is even possible to read a passage aloud fluently without understanding what was read. In contrast, in silent reading the primary goal is to comprehend and assimilate the meaning of the text. One does not naturally read something for the first time aloud, except in school or in the laboratory. In silent reading one can comprehend the text from just a subset of key words. They may also differ in the degree to which they invoke grapheme to phoneme decoding; in silent reading it would be possible to use a mechanism which decoded grapheme to semantic meaning without going through a phonemic step. In oral reading, the phonemic level must eventually be accessed for speech output.

We hypothesize that normal readers use different strategies in silent and oral reading. During silent reading they may use context cues to help decode single words, skip words while still grasping the mean- 
ing, and to some extent they may avoid the graphemeto-phoneme step. In oral reading these "sophisticated reading processes" are less relevant and, therefore, may be less used, since each word must be dealt with anyway, at the lowest level, phoneme by phoneme. We hypothesize that the normal readers' increase in theta has to do with some aspect of this change in strategy.

We further hypothesize that the dyslexics tend to use a mixture of word-by-word and context-cue strategies in both silent and oral reading, although they are deficient in both strategies. There are several arguments to support this hypothesis. Our dyslexics performed very poorly on the nonsense-word subtest of the reading subskills battery (Yingling and Galin 1982), and Myklebust (1968) found that this subtest was the most effective one in separating his dyslexics from controls. This indicates that the dyslexics can make at least some use of context and other "top-down" cues in decoding individual words, and that they do use these cues; when there is no context to support the grapheme-to-phoneme translation they do poorly. It seems plausible to suppose that the dyslexics use whatever top-down cues they have mastered in order to compensate; in oral reading they may be invoking context cues more than the normals do. We do not have direct evidence that in silent reading they use context less than the normal readers, but this inference is suggested by our findings that in narrative speech they show significant deficits in coherence and use significantly shorter communication units (Davenport et al. 1985).

This formulation would explain why the dyslexics' reading speed did not change much from silent to oral. It is also congruent with the finding that their theta power does not change as much as the normals' from silent to oral, and is slightly higher than the normals' during RS, and slightly lower during RO. If this interpretation is correct, then the difference between groups in theta may not be a specific index of the underlying deficit in the dyslexics. It would be simply a sign that they are not using the same strategy as the normal readers, but not a sign of what keeps them from using it.

The difference between groups in the low beta band appeared to be related to some aspect of overt speech. In a previous paper (Davenport et al. 1985), we reported two distinct deficits in these childrens' narrative speech performance. They used shorter communication units (independent clauses with all their modifiers), and a higher percentage of their words were non-communications (words which are extraneous to the speaker's intended meaning). The two measures were uncorrelated; they relate to different aspects of effectiveness in expression. Rate of speech was the same for both groups. It was not possible to distinguish whether the dyslexics' difficulty in expressing themselves was due to problems in word-finding, or in converting ideas into sentences, or due to confusion in the ideas themselves. However, in oral reading the organization of ideas and the arrangement of the ideas in sentences is already handled. Therefore, if the dyslexics' smaller increase in low beta in the "talking" tasks is related in some way to their performance deficits in narrative speech, the low beta is more likely to be related to word-finding.

It is unlikely that the theta and low beta effects are due to muscle activity and tongue movement accompanying speaking aloud. EMG would be greatest in the higher frequency bins, and our low beta effect was greatest at the low end of the band. It seems even more implausible that EMG "contamination" could have produced a group difference in the theta band and not in the alpha band.

\section{Important negative results}

\section{(1) No group differences in alpha}

Because of our work with alpha asymmetry in cognitive tasks we were particularly interested in alpha during the dyslexics' reading. In a previous study (Galin et al. 1988), we had not found any support for the "abnormal lateralization" theories of dyslexia (for reviews, see Kinsbourne and Hiscock 1981; Hiscock and Kinsbourne 1982). We had used the change in alpha asymmetry from speaking to block design construction as a measure of lateral specialization, and found that our severely dyslexic children showed the same taskdependent asymmetry of EEG alpha as the normal readers. Nevertheless, it was possible that the purported differences in lateralization relevant to dyslexia might only show up during reading. However, the results of the present analysis are clear: the dyslexics did not significantly differ from the normal readers in alpha power or in the direction or degree of asymmetry, at any lead, in either cohort, regardless of type of reading or level of difficulty. While there were many differences between the tasks in alpha power and alpha asymmetry, consistent with our previous findings (Galin et al. 1982), there were no effects in the alpha band related to group.

\section{(2) No group differences in asymmetry}

The group effects in theta and beta showed no interaction with SIDE. It is possible that use of the linked-ears reference may have prevented seeing an asymmetry in the group differences. This hypothesis could be tested by repeating the tasks with references to the ispilateral ear, or to a midline non-cephalic reference. However that may be, in the data at hand we find no support for theories of dyslexia based on abnormalities of lateral specialization, in theta and 
beta as well as alpha, even when recordings are made during reading.

\section{(3) No difficulty effects}

The absence of consistent effects related to the difficulty of the reading materials is puzzling. However, difficulty is a complex construct, and it may be affected by many different dimensions of task demand: the types of processes, how many are called for, and the rate of processing required (Galin et al. 1978). Thus, oral and silent texts judged to be 2 grades above a child's reading level may be "difficult" in different ways. Also, the particular task demand which is limiting may differ among different children. The individual's response to difficulty may also vary, e.g., a child may choose to slow down to preserve comprehension, or sacrifice comprehension to preserve speed. These variables should be controlled in future research.

The UCSF dyslexia project: screening, replication, task selection

Three features distinguish this project from many previous dyslexia studies. The first is that we placed great emphasis on recruiting relatively "pure" dyslexics. We selected children with severely impaired reading who were free from other conditions which a priori could lead to reading failure as a secondary symptom, or which could produce EEG abnormalities by themselves and thus confound the interpretation of any positive findings. Only a small fraction of potential subjects initially contacted was accepted. It is important to stress that the rejection rate was as frustratingly high for control candidates as for dyslexic candidates. In many studies the controls have not been given the same full screening as the index cases. The stringency of our selection procedure allows us to infer with some confidence that our findings are correlates of dyslexia per se and not due to unrelated disabilities also present by chance. On the other hand, our sample is not at all representative of the spectrum of children with learning disabilities encountered in the typical classroom or remedial clinic, and generalization of our findings to other more heterogeneous populations must be cross-validated.

The second distinguishing feature of our project is the 2-cohort design which permits cross-validation of any first cohort findings on the independently gathered second cohort. This proved to be quite important, because we encountered many instances where group differences with significance values of $P<0.01$ and even $P<0.001$ were found in one cohort which did not replicate, in spite of the great care which we took to insure the equivalence of the two samples.

The third feature of our study is that we recorded while the children were reading, both silently and orally, at two levels of difficulty, including a condition which was relatively easy for the dyslexics and one which was relatively hard for the normal readers. Natural reading (extracting meaning from connected prose) is a complex of many subprocesses. In addition to the decoding of individual words or syllables, it requires targeted eye movements, the integration of serial fixations, making use of transition probabilities between words, and the understanding of syntax. Because "natural" behaviors are so complex, they are more difficult to control than the fragments of behavior usually used in the laboratory (e.g., reading single words, or nonsense syllables). Nevertheless, because of the wide range of cognitive demands presented by our reading conditions, plus the listening and narrative speech tasks, we were able to isolate certain gross dimensions of the tasks, e.g., by comparing those with talking vs. those with no talking, reading vs. no reading. We do not know of any other EEG studies of talking other than our own. These results have provided clues as to which specific subprocesses should be isolated and studied in more detail in future research.

\section{Other studies of dyslexics' EEGs during reading}

There have been remarkably few studies of dyslexia which employed spectral analysis of EEG recorded during reading (Sklar et al. 1973; Rebert et al. 1978; Duffy et al. 1980, 1988; Leisman and Ashkenazi 1980; Lubar and Deering 1981; Duffy and McAnulty 1985). All of these studies examined silent but not oral reading and did not control or manipulate difficulty for the individual subject. Sklar et al. (1973) reported that dyslexics $(n=12)$ had lower relative alpha power at rest, and greater relative theta power than the controls $(\mathrm{n}=13)$ at rest and during reading. In their data, the rest condition difference discriminated the groups better than the reading condition difference. We have discussed some problems associated with the use of relative power measures in a previous paper (Fein et al. 1986). Leisman and Ashkenazi (1980) report no difference between groups during reading, but lower relative alpha power in dyslexics during rest. Duffy et al. (1980) reported higher absolute alpha and theta power in dyslexics, with greater group differences during reading and memorizing nonsense forms than during rest. However, this study included only 8 dyslexics, only 4 right handed. A subsequent study with 30 dyslexics divided into 3 subtypes found differences only in delta and low beta (Duffy et al. 1988). Rebert et al. (1978) studied children characterized as "general learning disability with reading difficulty," with and without severe oral language problems, which he termed "dyslexic" and "dysphasic." There was no normal control group. He found decreases in temporal and parietal theta power during listening, reading, and 
drawing compared to resting (linked ears). Lubar and Deering (1981) studied a mixed group of 34 learning disabled children at rest and during reading and other tasks with bipolar leads F7-T5 and F8-T6. No statistical evaluation of group or task differences is reported, but it appears that in general the LD children showed less alpha and more delta and theta than the normals. For the normals theta and low beta increased in reading.

A recent very sophisticated series of studies by Pfurtscheller and colleagues have examined the timecourse of changes in alpha power and distribution (125 msec intervals) during silent reading of single words (Pfurtscheller and Klimesch 1989). Unfortunately this work so far has been limited to adults, normal readers, single concrete nouns, and the alpha band, but their approach could be extended to address the findings we have reported here.

In summary, we have studied a very pure sample of dyslexic and normal readers, under passive conditions and in a wide range of active cognitive tasks. It was not until we examined the EEG while the children were actually reading that we found large, replicable differences between the dyslexics and normal readers. In spite of the task specificity of these findings, we cannot yet say that these effects are related to the neurological deficits underlying the dyslexia; it is possible that they represent only compensatory or secondary cognitive consequences of the underlying deficit. In addition to the group differences, we found unexpected task differences in spectral power between oral and silent reading, speaking and listening, which were unrelated to group. These results suggest that theta and low beta activities in the temporal lobe are related to high level language processes. Further studies are needed to clarify the functional significance of this activity and the physiological mechanisms generating it.

\section{References}

Ahn, H., Prichep, L., John, E.R., Baird, H., Trepetin, M. and Kaye, $\mathrm{H}$. Developmental equations reflect brain dysfunctions. Science, 1980, 210: 1259-1262.

Bakker, D.J. and Vinke, J. Effects of hemisphere-specific stimulation on brain activity and reading in dyslexics. J. Clin. Exp. Neuropsychol., 1985, 7: 505-525.

Benton, A.L. Developmental dyslexia: neurological aspects. In: W.J. Friedlander (Ed.), Advances in Neurology. Vol. 7. Current Reviews of Higher Nervous System Dysfunction. Raven Press, New York, 1975.

Callaway, E., Halliday, R. and Naylor, H. Hyperactive children's event-related potentials fail to support underarousal and maturational lag theories. Arch. Gen. Psychiat., 1983, 40: 1243-1248.

Cohen, J. Statistical Power Analysis for the Behavioral Sciences, revised edition. Academic Press, New York, 1977.

Coltheart, M., Patterson, K. and Marshall, J. (Eds.). Deep Dyslexia. Rutledge, Kegan and Paul, London, 1980.
Conners, C.K. Critical review; EEG and neurophysiological studies in dyslexia. In: A.L. Benton and D. Pearl (Eds.), Dyslexia: an Appraisal of Current Knowledge. Oxford University Press, New York, 1978: 251-261.

Davenport, L., Yingling, C.D., Fein, G., Galin, D. and Johnstone, J. Narrative speech deficits in dyslexics. J. Clin. Exp. Neuropsychol., 1985, 8: 347-361.

Davidson, R.J., Saron, C., Leslie, S.C. and Reiner, P.D. Visual evoked response estimates of interhemisphere transfer time: comparison of dyslexics and normals. J. Clin. Exp. Neuropsychol., 1985, 7: 634.

Doyle, J., Ornstein, R.E. and Galin, D. Lateral specialization of cognitive mode: an EEG study. Psychophysiology, 1974, 11: 567578.

Duffy, F.H. and McAnulty, G.B. Brain electrical activity mapping (BEAM): the search for a physiological signature of dyslexia. In: F.H. Duffy and N. Geschwind (Eds.), Dyslexia: a Scientific Approach to Clinical Evaluation. Little, Brown and Co., Boston, MA, 1985

Duffy, F.H., Denckla, M.B., Batels, P.H., Sandini, G. and Keissling, L. Dyslexia: automated diagnosis by computerized classification of brain electrical activity. Ann. Neurol., 1980, 7: 421-428.

Duffy, F.H., Denckla, M.B., McAnulty, G.B. and Holmes, J. Neurophysiological studies of dyslexia. In: F. Plum (Ed.), Language, Communication, and the Brain. Res. Publ. Ass. Nerv. Ment. Dis., 1988,66 .

Fein, G., Galin, D. Yingling, C.D., Johnstone, J., Davenport, L. and Herron, J. EEG spectra in dyslexic and control boys during resting conditions. Electroenceph. clin. Neurophysiol., 1986, 63: $87-97$.

Galin, D., Johnstone, J. and Herron, J. Effects of task difficulty on EEG measures of cerebral engagement. Neuropsychologia, 1978, 16: $461-472$.

Galin, D., Ornstein, R., Herron, J. and Johnstone, J. Sex and handedness differences in EEG measures of hemispheric specialization. Brain Lang., 1982, 16: 19-55.

Galin, D., Herron, J., Johnstone, J., Fein, G. and Yingling, C. EEG alpha asymmetry in dyslexics during speaking and block design tasks. Brain Lang., 1988, 35: 241-253.

Gates, A.I. and Macginitie, W.H. Gates-MacGinitie Reading Tests. Teachers College Press, New York, 1965.

Gray, W.F. Gray Oral Reading Test. Bobbs-Merrill, Indianapolis, IN, 1963.

Hiscock, M. and Kinsbourne, M. Laterality and dyslexia: a critical view. Ann. Dyslexia, 1982, 32: 177-228.

Hughes, J.R. EEG and neurophysiological studies in dyslexia. In: A.L. Benton and D. Pearl (Eds.), Dyslexia: an Appraisal of Current Knowledge. Oxford University Press, New York, 1978: 251-261.

Huynh, H. and Feldt, L.F. Estimation of Box correction for degrees of freedom for sample data in randomized block and split plot designs. J. Ed. Statist., 1976, 1: 69-82.

John, E.R., Karmel, B.Z., Corning, W.C., Easton, P., Brown, P., Ahn, H., Harmony, T., Prichep, L., Toro, A., Gerson, I., Bartlett, F., Thatcher, R., Kaye, H., Valdes, P. and Schwartz, E. Neurometrics. Science, 1977, 196: 1393-1410.

John, E.R., Ahn, H., Prichep, L., Trepetin, M., Brown, D. and Kaye, H. Developmental equations for the EEG. Science, 1980, 210: $1255-1258$.

Johnstone, J., Galin, D., Fein, G., Yingling, C.D., Herron, J. and Marcus M. Regional brain activity in dyslexic and control children during reading tasks: visual probe event-related potentials. Brain Lang., 1984, 21: 233-254.

Just, M. and Carpenter, P. Psychology of Reading and Language Comprehension. Allyn Publ., New York, 1986.

Kinsbourne, M. and Hiscock, M. Cerebral lateralization and cognitive development. Conceptual and methodological issues. In: G.W. 
Hynd and J.E. Obrzut (Eds.), Neuropsychological Assessment and the School-Age Child: Issues and Procedures. Grune and Stratton, New York, 1981.

Klass, D. and Bickford, R.G. Glossokinetic potentials appearing in the EEG. Electroenceph. clin. Neurophysiol.. 1960), 12: 239.

Leisman. G. and Ashkenazi. M. Aetiological factors in dyslexia. IV. Cerebral hemispheres are functionally equivalent. Neuroscience. 1980. 11: 157-164

Lubar, J.F. and Deering. W.M. Behavioral Approaches to Neurology. Academic Press. New York. 1981.

Myklebust. H.R. Progress in Learning Disabilities. Grune and Stratton. New York, 1968.

Pfurtscheller. G. and Klimesch, W. Cortical activation pattern during reading and recognition of words studied with dynamic event-related desynchronization mapping. In: K. Maurer (Ed.), Topographic Brain Mapping of EEG and Evoked Potentials. Springer. Berlin. 1989: 303-313.

Rebert, C.S. Wexler, B.N. and Sproul, A. EEG asymmetry in educationally handicapped children. Electroenceph. clin. Neurophysiol. 1978. 45: 436-442
Rosenthal, R. Meta-Analytic Procedures for Social Research. Applied Social Research Methods Series, Vol. 6. Sage Publications. Beverly Hills, CA, 1984.

Sklar, B. Hanley, J. and Simmons, W.W. A computer analysis of FEG spectral signatures from normal and dyslexic children. IEEE Trans. Biomed. Eng., 1973, 20: 20-26.

Sperling. M.R. and Engel, Jr.. J. EEG recording from the temporal lobes: a comparison of ear, anterior temporal and nasopharyngeal electrodes. Ann. Neurol., 1985, 17:510-513.

Fouwen. B.C.L. and Prechtl, H.F.R. The neurological examination of the child with minor nervous dysfunction. Topics Dev. Med., 1970. 30: $1-105$.

Wechsler. D. Wechsler Intelligence Scale for Children-Revised (WISC-R). The Psychological Corporation. New York, 1974.

Yingling, C.D. and Galin, D. Neuropsychology of reading disorders. Final report: NICHD Contract N01-HD-8-2824, University of California. San Francisco. CA.1982.

Yingling, C.D.. Fein, G.. Galin, D., Peltzman, D. and Davenport, L. Neurometrics does not detect "pure" dyslexics. Electroenceph. clin. Neurophysiol. 1986, 63: 426-430). 